\title{
Computer-assisted pattern recognition model for the identification of slowly growing mycobacteria including Mycobacterium tuberculosis
}

\author{
Brian D. Plikaytis, ${ }^{1 *}$ Bonnie B. Plikaytis ${ }^{2}$ and Thomas M. ShinnicK ${ }^{2}$ \\ Biostatistics and Information Management Branch ${ }^{1}$ and Hansen's Disease Laboratory, Respiratory Diseases Branch ${ }^{2}$, \\ Division of Bacterial and Mycotic Diseases, National Center for Infectious Diseases, Mailstop C09, Centers for Disease \\ Control, Atlanta, GA 30333, USA
}

(Received 27 April 1992; revised 6 July 1992; accepted 20 July 1992)

\begin{abstract}
We present a computerized pattern recognition model used to speciate mycobacteria based on their restriction fragment length polymorphism (RFLP) banding patterns. DNA fragment migration distances were normalized to minimize lane-to-lane variability of band location both within and among gels through the inclusion of two internal size standards in each sample. The computer model used a library of normalized RFLP patterns derived from samples of known origin to create a probability matrix which was then used to classify the RFLP patterns from samples of unknown origin. The probability matrix contained the proportion of bands that fell within defined migration distance windows for each species in the library of reference samples. These proportions were then used to compute the likelihood that the banding pattern of an unknown sample corresponded to that of each species represented in the probability matrix. As a test of this process, we developed an automated, computer-assisted model for the identification of Mycobacterium species based on their normalized RFLP banding patterns. The probability matrix contained values for the $M$. tuberculosis complex, $M$. avium, $M$. intracellulare, $M$. kansasii and $M$. gordonae species. Thirty-nine independent strains of known origin, not included in the probability matrix, were used to test the accuracy of the method in classifying unknowns: 37 of $39(94.9 \%)$ were classified correctly. An additional set of 16 strains of known origin representing species not included in the model were tested to gauge the robustness of the probability matrix. Every sample was correctly identified as an outlier, i.e. a member of a species not included in the original matrix. This strategy may be readily adapted to other chromatographic and electrophoretic systems that generate peak profiles or banding patterns.
\end{abstract}

\section{Introduction}

Techniques such as DNA fingerprinting, ribotyping and restriction fragment length polymorphism (RFLP) analyses have been used to investigate the genetic relatedness of bacterial strains and species. Basically, each of these techniques generates a set of information about the genomic structure of the organism, typically a pattern of DNA fragment migration distances. The band migration distances are, in turn, used to describe similarities within a group of samples as well as to classify unknown strains. One difficulty inherent in each of these techniques is the comparison of a new pattern with previously collected patterns. This is due in part to variability in DNA migration distances from lane-to-lane within a gel and

* Author for correspondence. Tel. (404) 639-1364; fax (404) 6393970 .

Abbreviation: RFLP, restriction fragment length polymorphism. among gels. This variability can be minimized by normalization of the migration distances using internal standards of known size that are included in each sample. Kersters \& De Ley (1975) originally described a procedure for normalizing band migration distances on polyacrylamide gels after electrophoresis by using two bracketing internal standards and manipulating photographic negatives of the electrophoretic gels. Subsequently, Plikaytis et al. (1986) developed a mathematical system of normalizing these banding profiles which essentially algebraically described the technique of Kersters and De Ley, obviating the manual manipulation of photographic negatives and introducing a more objective approach to the normalization of electrophoregrams.

Normalized profiles can be compared with each other and with clusters of similar patterns generated using one of several procedures based on band migration distances or peak retention times. Jackman et al. (1983) detailed an 
early system for clustering electrophoregrams based on band migration distances. Kersters \& De Ley (1975) improved this technique by normalizing the electrophoregrams, and Plikaytis et al. (1986) developed an accurate method for clustering these patterns based on their normalized band or peak positions. These techniques usually generate a dendrogram illustrating the clustering and relatedness of a collection of strains.

To identify unknown strains, investigators will typically run the clustering programs on a collection of known samples, representing several characterized species of bacteria, intermixed with a small number of unknown specimens. The identification of the unknown strains will then be based on their location in the resulting dendrogram. This system is convincing if the clusters of different species in the dendrogram are well defined and separated. However, all too often this technique suffers in that it relies on subjective judgments made by the investigator, especially if the clusters contain some crossover among the species in the reference collection. That is, if the banding profiles are not normalized, the DNA band migration distances may contain significant lane-to-lane variability both across and among gels. This may result in the clusters not being well defined or containing members from different species, forcing the investigator to make judgmental determinations regarding the identification of the unknown strains. An automated system for comparing and classifying these patterns would help to eliminate the subjectivity inherent in the interpretation of these dendrograms. Additionally, an automated system would eliminate the subjective determinations involved with the simple visual comparison of patterns and would give the investigator the ability to examine several patterns simultaneously, a process that is tedious and prone to error when performed manually.

One computerized method that may be adapted for general pattern recognition is a numerical probabilistic classification scheme which was originally used to speciate bacteria given a series of biochemical test results (Dybowski \& Franklin, 1968; Sneath \& Sokal, 1973). This technique is based on a conditional probability model and was used by Dybowski \& Franklin (1968) to identify enterobacteria. S. P. Lapage and colleagues further developed the method to its most common implementation in a series of reports (Bascomb et al., 1973; Lapage et al., 1973; Willcox et al., 1973) and used it to create a discrimination procedure for strains of Gramnegative, aerobic, rod-shaped bacteria. Wayne et al. (1984) used these developments to derive a diagnostic probability matrix, which they used to classify slowly growing Mycobacterium species based on biochemical test results.

The present report describes how these procedures may be applied to RFLP data using DNA fragment migration distances. This process uses a library of normalized patterns derived from samples of known origin to create a probability matrix, which is then used to classify the patterns from samples of unknown origin. The probability matrix contains the proportion of bands that fall within defined migration distance windows for each species in the library of known samples. These proportions are then used to compute the likelihood that the DNA banding pattern for an unknown sample corresponds to the DNA banding pattern of each species represented in the probability matrix. As a test of this process, we developed an automated, computer-assisted model for the identification of Mycobacterium species based on their RFLP banding patterns.

\section{Methods}

Generation of RFLP patterns. The strains listed in Table 1 were used to create the initial frequency and probability matrices. Strains listed in Table 2 were used to test the specificity and robustness of the pattern recognition program. The sources, identification and propagation of these strains are described by Plikaytis et al. (1992). Standard biochemical tests and high-performance liquid chromatography of mycolic acids were used to determine the species of each strain tested (Butler et al., 1991). In addition, DNA probes were used to confirm the identification of the $M$. avium and $M$. intracellulare strains. Genomic DNA was isolated from these strains and their RFLP patterns generated as previously described (Plikaytis et al., 1992). A $\sim 1380 \mathrm{bp}$ fragment of the mycobacterial $h s p 65$ gene was amplified from chromosomal DNA using the polymerase chain reaction (Mullis \& Faloona, 1987). The amplified products were digested with the restriction enzymes BstNI and $X h o I$ in separate reactions. After digestion, $300 \mathrm{ng}$ of a $800 \mathrm{bp}$ DNA fragment and a $113 \mathrm{bp}$ DNA fragment were added, and the mixtures were electrophoresed on $6 \%$ polyacrylamide-Tris/borate/EDTA gels. The DNA bands were visualized by ethidium bromide fluorescence and photographed using Polaroid type 55 positive/negative film.

Normalization and combination of RFLP band patterns. The DNA band positions were recorded from the negatives using a Drawing Board II Digitizer (Cal Comp Digitizer Products Group, Scottsdale, Ariz., USA) interfaced with a Compaq Deskpro 386s/20 desktop computer. The band positions were recorded as digitizer-absoluteplotting-coordinates and converted to centimetres by calculating the simple distance between each band and the $800 \mathrm{bp}$ internal standard in each lane. Each band position was normalized to a fixed distance based on the position of the two internal standards as previously described (Plikaytis et al., 1986). Each RFLP banding pattern was scaled so that the $800 \mathrm{bp}$ and $113 \mathrm{bp}$ internal standards were positioned on 5.0 and $15.0 \mathrm{~cm}$, respectively, and the remaining bands were adjusted to range about them. Thus, lanes that ran slightly longer or shorter than normal were contracted or expanded, by a proportional amount, to a fixed distance using the following equation (Plikaytis et al., 1986):

$$
\mathrm{MD}_{\text {norm }}=5+\left[(15-5) \times\left(\mathrm{MD}-\mathrm{MD}_{800}\right)\right] /\left(\mathrm{MD}_{113}-\mathrm{MD}_{800}\right)
$$

where $\mathrm{MD}=$ the actual migration distance of the band to be normalized; $\mathrm{MD}_{113}$ and $\mathrm{MD}_{800}=$ the individual migration distances of the $113 \mathrm{bp}$ and $800 \mathrm{bp}$ internal standards, respectively, in the lane being normalized; and $\mathrm{MD}_{\text {norm }}=$ the final normalized migration distance. The RFLP patterns from the two enzyme digests were 
Table 1. Reference strains used to form the probability matrix

\begin{tabular}{|c|c|}
\hline Species & Strains \\
\hline M. avium & $\begin{array}{l}\text { TMC 716, TMC 724, TMC 1461, TMC 1464, 86-8751, 86-8813, 86-8810, 88-1107, 89-615, 89-1177, 89-1179, 90-9030, 90-9116, } \\
\text { LR541, LR542, LR551, LR588 }\end{array}$ \\
\hline M. bovis $\mathrm{BCG}$ & TMC 1013, TMC 1024 (Glaxo), Rosenthal \\
\hline M. gordonae & $\begin{array}{l}\text { TMC 1324, TMC 1325, 85-736, 86-920, 86-1085, 87-613, 87-840, 87-1158, 88-329, 88-358, 88-404, 88-405, 88-1012, 88-1075, } \\
88-1160,89-467,89-676,89-1183,91-343,91-403,91-406,91-428,91-537,91-575,91-598,91-638,91-691,91-637,91-652, \\
91-688,91-689\end{array}$ \\
\hline M. kansasii & $\begin{array}{l}\text { TMC 1204, ATCC 12478, 85-961, 85-983, 85-1010, 85-1069, 89-304, 89-344, 89-366, 89-367, 89-368, 89-371, 89-374, 89-492, } \\
\text { 89-537, 89-538, 89-1361, 90-4343, 91-348, 91-450, 91-540, 91-543, 91-573, 91-627, 91-771, 91-4654, 91-IT-197, 91-IT-923, } \\
\text { 91-IT-1029, 91-IT-1071 }\end{array}$ \\
\hline M. tuberculosis & H37Ra, T5, T6, T12, 8112, 8113, 8114, 8118, 8125, 8126, 8129, 8136, 8137, 8138, 8140, 8144, 8145, 8147, 8148 \\
\hline
\end{tabular}

Table 2. Tests strains used to measure the accuracy of identification using the probability matrix

\begin{tabular}{|c|c|}
\hline Species & Strains \\
\hline M. africanum & TMC 5122 \\
\hline M. avium & $86-8647,88-1146,89-638,89-681,90-9083,90-9103,91-9010,91-9019$, LR25 \\
\hline M. chelonae & TMC 1524,801356 \\
\hline M. fortuitum & TMC 1530, TMC 1547 \\
\hline M. gordonae & $86-703,87-808,88-1159,89-516,89-1113,91-403,91-690$ \\
\hline M. intracellulare & $88-1152,88-1314,89-867,89-1049,90-1123,91-306$ \\
\hline M. kansasii & $85-830,89-367,89-593,89-1121,90-$ IT-942, 91-IT-1055, 91-452 \\
\hline \multicolumn{2}{|r|}{ 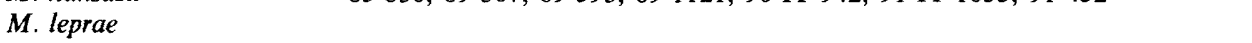 } \\
\hline M. marinum & TMC 1218 \\
\hline M. nonchromogenicum & TMC 1481 \\
\hline M. phlei & TMC 1548 \\
\hline M. scrofulaceum & TMC 1309, TMC 1321, 2197-4-84 \\
\hline \multicolumn{2}{|l|}{ M. simiae } \\
\hline M. smegmatis & TMC 1533 \\
\hline M. szulgai & $91-698$ \\
\hline M. tuberculosis & $\begin{array}{l}90-8003,90-8087,90-8091,90-8099,90-8188,91-8005,91-8024,91-8144, \\
91-8255\end{array}$ \\
\hline M. vaccae & TMC 1526 \\
\hline$M$. xenopi & 80184 \\
\hline
\end{tabular}

combined by adding a fixed constant, $20 \mathrm{~cm}$, to the normalized migration distances of the BstNI pattern and then merging these distances with the $X h o I$ pattern. In this way, there was no overlap of bands between the two patterns, and one large profile was constructed combining the two digests yet retaining the identity of each pattern. The band migration distances within the combined patterns ranged from 0.0 to $39.0 \mathrm{~cm}$.

Formation of the frequency-probability matrices. The 0.0 to $39.0 \mathrm{~cm}$ migration distance range was split into 26 equally spaced distance windows, each $1.5 \mathrm{~cm}$ wide. This window corresponds to an $\sim 4.5 \mathrm{~mm}$ window on the original photograph. The frequency matrix is composed of 26 rows, corresponding to the 26 distance windows, and five columns, corresponding to each species or complex group in the collection of reference isolates. Each frequency matrix entry tabulates the number of times one or more bands from the normalized profile for each reference isolate within a given species falls within the defined window. The probability matrix is formed by dividing the frequency counts in each window for each species or complex group by the total number of isolates in that species or group. Thus, this matrix describes the proportion of times that one or more bands fell within a certain distance window for the reference strains of each species. This proportion also describes the probability of observing one or more bands within a given distance window for an isolate of a given species.

A problem arises with probability matrix entries containing the absolute probabilities of 0.0 and 1.0 , representing, respectively, windows which do not include bands from any strains in the reference collection for a given species and windows which include bands from all the strains. This is undesirable in that it associates an assignment of an unconditional probability based on a limited sample of strains. Also, using 0.0 and 1.0 in the probability matrix results in the multiplication by zero in subsequent calculations, leading to trivial results. Laplace's law of succession (Willcox et al., 1973) was used to calculate the probabilities in these instances. The probability is defined as the ratio $(m+1) /(n+2)$, where $m=$ the number of times one or more bands fall 
Table 3. Representative likelihood calculations illustrating the use of the probability matrix

Probabilities are expressed in terms of percentages (i.e. probabilities $\times 100$ ).

\begin{tabular}{ccccc}
\hline \hline Distance window & Species 1 & Species 2 & Species 3 & Unknown strain* \\
\hline 1 & 99 & 99 & 1 & + \\
2 & 99 & 99 & 1 & + \\
3 & 1 & 99 & 95 & - \\
4 & 1 & 1 & 99 & - \\
5 & 35 & 99 & 99 & + \\
\hline
\end{tabular}

Maximum possible likelihood:

Species $1=0.99 \times 0.99 \times(1.0-0.01) \times(1.0-0.1) \times(1.0-0.35)=0.6244$

Species $2=0.99 \times 0.99 \times 0.99 \times(1.0-0.99) \times 0.99=\quad 0.9510$

Species $3=(1.0-0.01) \times(1.0-0.01) \times 0.75 \times 0.99 \times 0.99=\quad 0.7205$

The likelihood that the unknown strain belongs to each species:

Species $1=0.99 \times 0.99 \times(1.0-0.01) \times(1.0-0.01) \times 0.35=$

Species $2=0.99 \times 0.99 \times(1.0-0.99) \times(1.0-0.01) \times 0.99=$

Species $3=0.01 \times 0.01 \times(1.0-0.75) \times(1.0-0.99) \times 0.99=$

Total likelihood:

\begin{tabular}{c}
0.3362 \\
0.0096 \\
$2.475 \times 10^{-7}$ \\
\hline 0.3458
\end{tabular}

Normalized likelihood:

$0 \cdot 3362 / 0 \cdot 3458=$

$0 \cdot 0096 / 0 \cdot 3458=$

0.9722

$2.475 \times 10^{-7} / 0 \cdot 3458 \approx$

0.0278

$0 \cdot 000$

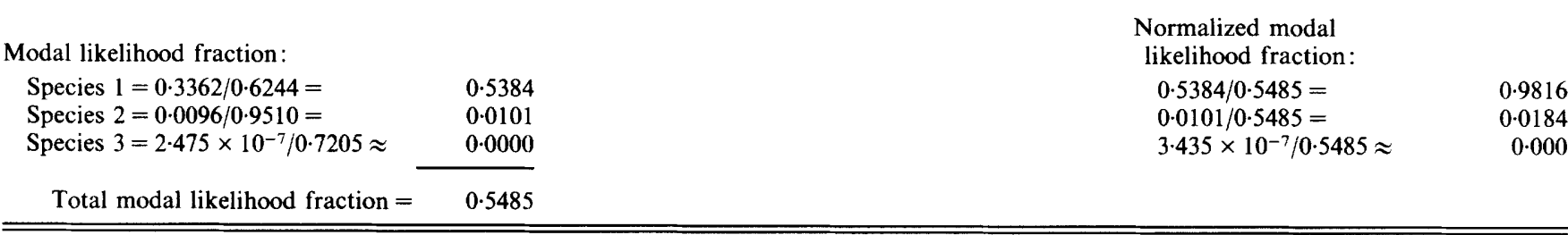

* Results from a typical unknown strain. (+) indicates one or more bands fall within a given distance window; $(-)$ indicates the absence of a band.

within a given window and $n=$ the number of strains in the reference collection representing the species in question.

Calculation of likelihood and identification scores. The calculation of likelihood and identification scores is based on the application of a conditional probability model which determines the likelihood that an unknown strain is a member of any species in the probability matrix (Willcox et al., 1973). These calculations are detailed in Table 3. To illustrate the calculations involved in the pattern recognition model, Table 3 contains a probability matrix generated for patterns of three hypothetical species and a pattern for an 'unknown' strain. This probability matrix indicates the proportion of hypothetical reference strains that contained one or more bands within a given distance window and species. For example, $35 \%$ of species 1 strains possessed bands that fell in distance window number 5 . Each matrix entry describes the probability of observing a band within a given window. Assume for this example that each species is represented by a sample of 100 strains so that matrix entries of 1 correspond to those distance windows where none of the strains had bands and matrix entries of 99 indicate that all strains for the given species had at least one band fall within the specified window. The last column of Table 3 contains the pattern of a hypothetical 'unknown' specimen in which $(+)$ indicates that one or more bands fell within a given distance window and (-) indicates the absence of a band.

An ideal reference strain for a given species would contain a band in all distance windows where the probability exceeds 0.50 (or $50 \%$ ) and lack a band in all distance windows where the probability is less than $0 \cdot 50$. Thus, the maximum possible likelihood for a particular species is the probability that the ideal reference strain represents that species. This quantity is calculated by taking the product of the probabilities in the matrix that describe the species (Table 3). For those windows where the probability exceeds 0.50 , the probability is abstracted from the matrix directly. For windows where the matrix entry is less than 0.50 , the probability is taken to be ( 1.0 minus the probability represented by the matrix entry).

The likelihood that the unknown strain is a member of one of the species represented in the frequency-probability matrix is the probability that the normalized migration distances for the bands of an isolate from that species will fall in the same distance windows as the bands from the unknown isolate. The likelihood for each species is found by multiplying the probabilities associated with each distance window for that species. When the unknown strain contains a band within a window, the probability is abstracted from the matrix directly. The probability of not observing a band within a window [windows with ( $(-)$ results] is simply ( 1.0 minus the probability represented by the matrix entry). Table 3 illustrates these calculations for the sample unknown strain for each of the three hypothetical species.

The value of a likelihood is normalized by dividing it by the sum of the likelihoods for all species. These normalized values are referred to as identification scores and will range from 0.0 to 1.0 . Ideally, the scores should be close to 1.0 for the true species of the unknown sample and much less than 1.0 for the remaining species in the probability matrix.

Calculation of modal likelihood fraction. One limitation of this approach is that a normalized score close to 1.0 is still possible in some instances in which the unknown strain belongs to a species not represented in the probability matrix. Dybowski \& Franklin (1968) attempted to solve this problem by creating a quantity they termed the modal likelihood fraction. This quantity is calculated by dividing the likelihood for an unknown strain being of a given species by the maximum possible likelihood for a strain from that species (i.e. the likelihood for the idealized strain of a given species). Strains from 
species not represented in the probability matrix should exhibit a low modal likelihood fraction. These modal likelihood fractions are then normalized by dividing each by the sum of the modal likelihood fractions for all species (Table 3).

Computations. The computations were performed in a software package using Microsoft QuickBASIC, version 4.5 (Microsoft) on a Compaq Deskpro 386s/20 desktop computer. Copies of the program may be requested from the corresponding author.

\section{Results}

\section{Probability matrix and typical calculations}

Recently, we reported a rapid two-step assay to detect and identify slow-growing Mycobacterium species including $M$. tuberculosis (Plikaytis, et al., 1992). The first step of this rapid procedure uses the polymerase chain reaction to amplify a 1380 bp portion of the $h s p 65$ gene from all Mycobacterium species. The hsp65 gene, which encodes a $65000 \mathrm{Da}$ heat shock protein, was chosen as the target for amplification because it is highly conserved among all Mycobacterium species (Shinnick et al., 1987). The second step involves cleavage of the amplicons with the restriction enzymes $B s t \mathrm{NI}$ and $X h o \mathrm{I}$ to generate restriction fragment patterns that were characteristic of each species. In order to obtain a set of data that adequately describes the restriction fragment profiles for the reference strains, patterns were obtained for the 126 characterized and identified Mycobacterium strains listed in Table 1 . These included 17 strains of $M$. avium, 23 strains of $M$. intracellulare, 25 strains of the $M$. tuberculosis complex (M. tuberculosis, $M$. bovis, $M$. africanum), 30 strains of $M$. kansasii and 31 strains of $M$. gordonae.

The probability matrix generated from these data is shown in Table 4. Two groups of migration distances can be clearly seen. Windows representing 0.0 to $<20.0 \mathrm{~cm}$ correspond to the restriction fragment patterns from the $X h o \mathrm{I}$ digest while the windows representing $20 \cdot 0$ to $<39.0 \mathrm{~cm}$ correspond to the patterns from the $B s t \mathrm{NI}$ digest. The last two columns of Table 4 contain the results obtained with typical strains of the $M$. gordonae

Table 4. Probability matrix for five species of mycobacteria

Probabilities are expressed in terms of percentages (i.e. probabilities $\times 100$ ).

\begin{tabular}{|c|c|c|c|c|c|c|c|}
\hline \multirow[b]{2}{*}{$\begin{array}{l}\text { Distance window } \\
(\mathrm{cm})\end{array}$} & \multicolumn{5}{|c|}{ Probability of a window containing one or more bands for reference isolates of: } & \multirow[b]{2}{*}{$\begin{array}{c}\text { Unknown } \\
\text { strain } 1 \dagger\end{array}$} & \multirow[b]{2}{*}{$\begin{array}{l}\text { Unknown } \\
\text { strain } 2 f\end{array}$} \\
\hline & M. avium & M. intracellulare & M. kansasii & M. gordonae & $\begin{array}{l}\text { M. tuberculosis } \\
\text { complex* }\end{array}$ & & \\
\hline $0.0-<1.5$ & 5 & 4 & 3 & 3 & 4 & - & - \\
\hline $1.5-<3.0$ & 5 & 4 & 3 & 3 & 4 & - & - \\
\hline $3 \cdot 0-<4.5$ & 5 & 4 & 3 & 3 & 4 & - & + \\
\hline $4.5-6.0$ & 65 & 9 & 97 & 26 & 84 & - & - \\
\hline $9 \cdot 0-<10 \cdot 5$ & 5 & 91 & 3 & 3 & 4 & - & - \\
\hline $10 \cdot 5-<12 \cdot 0$ & 5 & 4 & 3 & 3 & 4 & - & - \\
\hline $12.0-<13.5$ & 5 & 4 & 3 & 3 & 96 & - & - \\
\hline $13 \cdot 5-<15.0$ & 5 & 4 & 3 & 3 & 4 & - & - \\
\hline $15 \cdot 0-<16.5$ & 5 & 4 & 3 & 3 & 4 & - & - \\
\hline $16 \cdot 5-<18.0$ & 5 & 4 & 3 & 3 & 4 & - & - \\
\hline $18 \cdot 0-<19.5$ & 5 & 4 & 3 & 3 & 4 & - & - \\
\hline $25.5-<27.0$ & 5 & 87 & 97 & 97 & 4 & + & - \\
\hline $27 \cdot 0-<28.5$ & 95 & 13 & 87 & 3 & 80 & - & - \\
\hline $28.5-<30 \cdot 0$ & 5 & 96 & 3 & 97 & 20 & + & + \\
\hline $30 \cdot 0-<31.5$ & 5 & 96 & 7 & 6 & 96 & - & + \\
\hline $31.5-<33.0$ & 95 & 13 & 7 & 94 & 96 & + & + \\
\hline $33 \cdot 0-<34.5$ & 95 & 96 & 97 & 74 & 96 & + & + \\
\hline $34 \cdot 5-<36 \cdot 0$ & 5 & 4 & 3 & 3 & 4 & - & - \\
\hline $36.0-<37.5$ & 5 & 4 & 3 & 3 & 4 & - & - \\
\hline $37 \cdot 5-<39 \cdot 0$ & 5 & 4 & 3 & 3 & 4 & - & - \\
\hline Number of strains & 17 & 23 & 30 & 31 & 25 & & \\
\hline
\end{tabular}

* M. tuberculosis complex includes $M$. tuberculosis, $M$. bovis and M. africanum.

$\dagger$ Results from a typical strain of the $M$. gordonae species. (+) indicates one or more bands fall within a given distance window; $(-)$ indicates the absence of a band.

$\ddagger$ Results from a typical strain of the $M$. chelonae species. 
Table 5. Typical likelihood calculations for an unknown strain from a species (M. gordonae) represented in the probability matrix

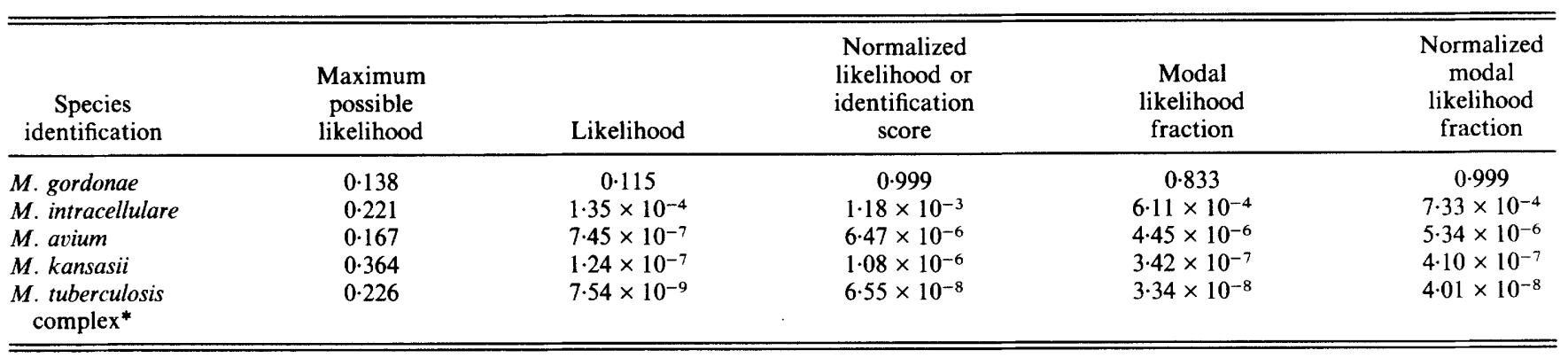

* M. tuberculosis complex includes $M$. tuberculosis, M. bovis and M. africanum.

Table 6. Typical likelihood calculations for an unknown strain from a species (M. chelonae) not represented in the probability matrix

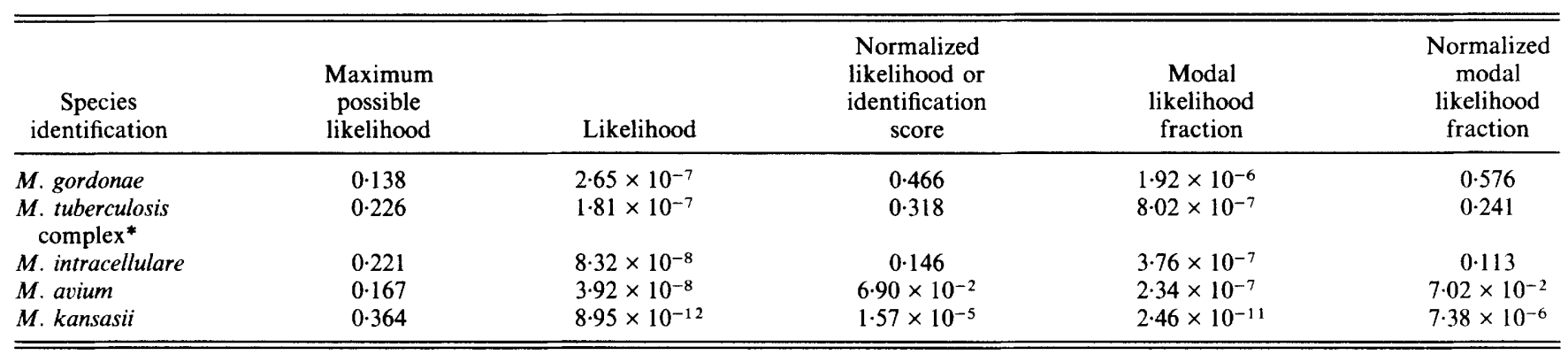

* M. tuberculosis complex includes $M$. tuberculosis, $M$. bovis and $M$. africanum.

(unknown strain 1) and $M$. chelonae (unknown strain 2) species, which were treated as unknown specimens and used to illustrate the likelihood calculations. In these columns, $(+)$ indicates that one or more bands fell within a given distance window; $(-)$ indicates the absence of a band.

To determine if an unknown strain produces a pattern similar to that of a characterized species, one compares the observed pattern of $(+)$ and $(-)$ windows with the sequence of probabilities for each species in the probability matrix. This is done by calculating the likelihoods and identification scores for the comparisons of the unknown with each species in the matrix as described in Methods. The results of these calculations for the 'unknown' strain 1 (M. gordonae) listed in Table 4 are shown in Table 5. Examining the normalized likelihood or identification score column in Table 5, it is easily seen that unknown strain 1 has a score close to the maximum possible identification score of 1.0 for $M$. gordonae ( 0.999$)$, and this score greatly exceeds the scores for the other species. This suggests strongly that unknown strain 1 is a member of the $M$. gordonae species. However, Dybowski \& Franklin (1968) observed that it is possible for unknown strains from species not included in the probability matrix to exhibit identification scores close to $1 \cdot 0$. To distinguish between these possibilities, one considers the modal likelihood frac- tions (the last two columns of Table 5). The modal likelihood fraction for $M$. gordonae, 0.833 , is also close to the maximum possible fraction, $1 \cdot 0$. This indicates that a strong correlation between the pattern of $(-)$ and $(+)$ results for the unknown and the cell entries for $M$. gordonae in the probability matrix, which are less than 0.50 and greater than 0.50 , respectively (Table 4 ). The high modal likelihood fraction together with the normalized modal likelihood fraction of 0.999 leads to the conclusion that the unknown strain is a member of the $M$. gordonae species. This conclusion is strengthened by the fact that each of these values is much greater than the corresponding calculations for the remaining Mycobacterium species.

Table 6 details similar calculations for unknown strain 2 (M. chelonae), which represents a species not included in the probability matrix. The likelihood that this strain belongs to any of the species represented in the probability matrix is extremely small, less than $1.0 \times 10^{-6}$ (Table 6, column 3). Although these likelihoods are small, the normalized likelihood or identification scores for $M$. gordonae and the $M$. tuberculosis complex, 0.466 and 0.318 , respectively, are moderately high. This reflects the fact that while the likelihoods that the strain is a member of the $M$. gordonae species or $M$. tuberculosis complex species are remote $\left(\leq 2.65 \times 10^{-7}\right)$, they are still greater than the likelihoods for the 
remaining species. This underscores the difficulty in relying solely on the identification scores to yield a definitive classification and emphasizes the need to calculate the modal likelihood fractions. In this example, the modal likelihood fractions for all species are extremely small, less than $1.0 \times 10^{-5}$, indicating little association between the pattern of $(-)$ and $(+)$ distance windows of the unknown and probability matrix entries for any of the species included in the library. The normalized modal likelihood fraction for $M$. gordonae is moderately high, as it was for the normalized likelihood. This is because the modal likelihood fraction for $M$. gordonae, while extremely small, is considerably greater than those of the remaining species in the table.

\section{Ability of the model to identify unknowns}

To test the ability of this pattern recognition program to identify unknown species, RFLP patterns were obtained from an additional 39 strains and subjected to this analysis. Importantly, (1) these test strains had been previously identified, although the identification code was not broken until after the analysis, (2) each test strain was a member of a species in the probability matrix, and (3) none of these 39 test strains were included in the library of reference strains used to form the probability matrix.

The following criteria were used to identify these 'unknowns' as a particular species: (1) the normalized likelihood or identification score and the normalized modal likelihood fraction must be $\geq 0.95$; and (2) the modal likelihood fraction must be $\geq 0 \cdot 10$.

Table 7 lists the results of the classification. Overall, the pattern recognition program correctly identified the species of $37(94.9 \%)$ of the 39 test strains. The two incorrectly classified strains, one strain each of $M$. intracellulare and $M$. kansasii, were classified as members of species not included in the probability matrix. The $M$. intracellulare strain had identification scores and normalized likelihood fractions greater than 0.99 for the correct species, but the modal likelihood fraction was less than $0 \cdot 1$; the $M$. kansasii strain had identification scores and normalized likelihood fractions less than 0.73 for the correct species, and the modal likelihood fraction was less than $0 \cdot 1$.

To test the robustness of this classification strategy, likelihood calculations were performed for 16 strains from 12 species not included in the probability matrix. These included two strains of $M$. chelonae, two strains of $M$. fortuitum, three strains of $M$. scrofulaceum, and one strain each of $M$. leprae, $M$. szulgai, $M$. smegmatis, M. phlei, M. marinum, M. vaccae, $M$. simiae, $M$. xenopi and $M$. nonchromagenicum. The identification scores and normalized modal likelihood fractions ranged from $0 \cdot 47$
Table 7. Results of classifying an independent collection of strains of mycobacteria

\begin{tabular}{lccc}
\hline \hline \multicolumn{1}{c}{ Species } & $\begin{array}{c}\text { No. of } \\
\text { strains }\end{array}$ & $\begin{array}{c}\text { No. } \\
\text { correctly } \\
\text { classified }\end{array}$ & $\begin{array}{c}\text { Percentage } \\
\text { correctly } \\
\text { classified }\end{array}$ \\
\hline $\begin{array}{l}\text { M. intracellulare } \\
\text { M. tuberculosis }\end{array}$ & 6 & 5 & $83 \cdot 3$ \\
$\quad$ complex* & 10 & 10 & 100 \\
$M$. avium & 9 & 9 & 100 \\
$M$. gordonae & 7 & 7 & 100 \\
$M$. kansasii & 7 & 6 & $85 \cdot 7$ \\
$\quad$ Total & 39 & 37 & $94 \cdot 9$ \\
\hline \hline
\end{tabular}

* M. tuberculosis complex includes $M$. tuberculosis, $M$. bovis and M. africanum.

to 0.98. However, in all cases the modal likelihood fractions were $\leq 3.05 \times 10^{-2}$. Based on the above criteria, each of these 16 strains would be correctly classified as being members of species not included in the probability matrix.

\section{Discussion}

The computer-based pattern recognition system described in this report is derived from a system used to classify bacteria based on their microbiological test results. As such, the method has been used and tested extensively for a variety of bacteria including mycobacteria (Bascomb et al., 1973; Friedman et al., 1973; Wayne et al., 1984). In essence, this system was extended to the recognition of RFLP patterns by characterizing the banding patterns as a series of probabilities that one or more bands would appear in user defined windows. Once lower limits for key likelihoods have been chosen, a quantitative method for comparing RFLP patterns emerges, which, in turn, allows an objective method for classifying unknown specimens through the use of a probability matrix. This method eliminates much of the subjectivity inherent in identifying unknown strains through other cluster analysis techniques where unknown strains are clustered with a collection of known strains. Such procedures suffer in the presence of poorly defined clusters where classification of unknown strains becomes more open to interpretation.

With RFLP patterns that include fewer than 10 bands it is usually an easy matter to visually compare the pattern of an unknown sample with patterns of known origin. This works well for a small number of samples. Visual comparison becomes unwieldy, however, when a large number of samples are compared against a large collection of known patterns. The system described in this report is fully automated and circumvents the subjective judgments inherent in other systems. 
Successful application of the technique described here requires the normalization of the RFLP band positions through the use of two internal standards. The use of internal standards greatly reduces the variability of band positions both within and across negatives (Plikaytis et al., 1986). Normalizing the banding patterns facilitates the creation of a more stable and descriptive probability matrix, and data from a variety of sources collected over lengthy periods of time may be used.

Many factors enter into the determination of the optimal distance window size used in the probability matrix. We normalized our RFLP banding patterns to range from 0.0 to $39.0 \mathrm{~cm}$ and attempted to select a window size small enough so that each window would contain a single band for a given species and large enough to bracket the band so that all like bands from the same species fell within the same window. The large number of matrix entries over $90 \%$ (Table 4) indicates that the chosen window size successfully bracketed like bands within each species. The windows also effectively excluded additional bands which were clearly different in size. However, in five instances $(M$. avium, 27.0 to $<28.5 \mathrm{~cm} ; M$. kansasii, 33.0 to $<34.5 \mathrm{~cm} ; M$. gordonae, 7.5 to $<9.0 \mathrm{~cm}$; and $M$. tuberculosis, 30.0 to $<31.5 \mathrm{~cm}$ and 33.0 to $<34.5 \mathrm{~cm}$ ) there were band doublets so close together that we could not separate them with a fixed window size. This results in a slight loss in RFLP information in that the $(+)$ results for these windows do not distinguish between the presence of one or two bands. Also, in a few cases, bands were distributed over two adjacent windows: $M$. intracellulare, 25.5 to $<27.0 \mathrm{~cm}(87 \%)$ and 27.0 to $<28.5 \mathrm{~cm}(13 \%) ; M$. gordonae, 24.0 to $<25.5(3 \%)$ and 25.5 to $<27.0(97 \%)$, and 30.0 to $<31.5(6 \%)$ and 31.5 to $<33.0(94 \%)$; and $M$. tuberculosis, 27.0 to $<28.5(80 \%)$ and 28.5 to $<30.0$ $(20 \%)$. The distance windows could have been adjusted individually to account for these problems, ensuring that all like bands were included within one window. However, this would introduce an element of subjectivity into the analysis and greatly complicate the analysis of patterns across all the species in the matrix. The overlap of these problem windows across the species in the matrix would also make this customization virtually impossible as the number of species in the probability matrix increases. Any new data or species may be easily accommodated with a system that employs a fixed window size.

The selection of 0.95 for the identification score and normalized modal likelihood fraction and $0 \cdot 10$ as the threshold value of the modal likelihood fraction used in rejecting an identification was based on empirical evaluation of the RFLP patterns. The closer the identification score, normalized modal likelihood fraction, and modal likelihood fraction are to $1 \cdot 0$, the more restrictive will be the identification. Thus, these numbers may be increased to make identifications more conservative and lowered to make them more liberal. The cost of making an incorrect identification when the criteria are too liberal (false positives) as opposed to rejecting a correct identification when the criteria are too conservative (false negatives) should dictate the magnitude of these numbers. As the number of cells in the probability matrix that contain probability values increases, i.e. as the number of bands in an RFLP pattern increases, the identification scores and normalized modal likelihood fraction may be safely increased and the modal likelihood fraction decreased. For comparison purposes, it may be noted that Wayne et al. (1984) used a value of 0.99 for their identification score and 0.01 as the threshold for the modal likelihood fraction. While their identification score was more conservative than the one used in this study, their cut-off for the modal likelihood fraction is more liberal than ours. Their choice of values was justified in that their probability matrix had many more cells containing probability values. In our study, an RFLP pattern had a maximum of nine bands which were reduced to seven distinct windows in the probability matrix for $M$. tuberculosis. The remaining species had fewer windows containing information. This small number of windows containing useful information dictated the use of a somewhat more liberal cut-off point for the identification scores and normalized modal likelihood fractions and a more conservative value for the modal likelihood fraction.

The chosen criteria resulted in the correct identification of 37 of the 39 test strains $(94.9 \%)$. The two strains that were not properly identified, an $M$. intracellulare strain and an $M$. kansasii strain, were classified as not belonging to a species included in the probability matrix based on their low modal likelihood fractions. This result is not too surprising given the strain-to-strain variation in the RFLP patterns within the $M$. intracellulare and $M$. kansasii clusters, even after the banding profiles were normalized (Plikaytis et al., 1992). That is, while the RFLP patterns of most of the reference strains of these two species shared similarities $>0.86$ (26 of $30 \mathrm{M}$. kansasii strains; 18 of $23 \mathrm{M}$. intracellulare strains), the RFLP patterns for the two incorrectly classified strains were similar at a lower level (0.75) to the other strains of their respective clusters (data not shown). Thus, the identification program may have difficulties in correctly identifying members of a species that display only modest similarity to the bulk of the reference strains that make up the probability matrix.

One potential limitation of this technique is the inability to conclusively identify an unknown strain as an outlier (Dybowski \& Franklin, 1968; Sneath \& Sokal, 1973); that is, determining that the strain is not a 
member of any species represented in the probability matrix. The use of $0 \cdot 1$ as the threshold for the modal likelihood fraction helps to circumvent this limitation. Indeed, all 16 test strains belonging to species not included in the probability matrix were correctly identified as outliers.

While the species included in our probability matrix comprise about $90 \%$ of the pathogenic mycobacterial isolates and $>86 \%$ of all mycobacterial isolates recovered in public health laboratories in the United States (Good \& Snider, 1982), future studies will expand the probability matrix to include more species. Indeed, one attractive feature of the probability matrix and pattern recognition methodology is the ability to include as many new species or subspecies as desired as the data become available to describe the RFLP patterns for the new groups of strains. The probability matrix may also be strengthened by the inclusion of additional strains of existing species. This will lead to more accurate estimates of the probability values. It may also be possible to improve the identification power of the program by separating the patterns of certain species into two or more columns. That is, although the RFLP pattern of each strain of a given species displays greater similarity to the patterns of all other strains of that species than to the patterns of strains from other species, there may be sufficient strain-to-strain variation in the RFLP patterns within a species that strains may fall into two or more closely related subclusters. This is particularly true for species known to contain defined subspecies such as $M$. chelonae subspecies chelonae and subspecies abscessus. This situation may easily be accommodated by separating a $M$. chelonae column in the probability matrix into two columns representing the two subspecies.

The automated, computer-assisted strategy for identifying Mycobacterium isolates using normalized RFLP banding patterns described in this article may be readily adapted to other chromatographic and electrophoretic systems which generate peak profiles or banding patterns such as DNA fingerprinting, ribotyping, highperformance liquid chromatography (HPLC), gas-liquid chromatography (GLC) and polyacrylamide gel electrophoresis (PAGE). Given a library of reference strains, probability matrices could be created for each of these applications and used to identify future unknown samples. For example, after implementing the normalization procedures described by Plikaytis et al. (1986), PAGE patterns may be treated in the same manner as the RFLP patterns described in this report. HPLC and GLC peak retention or elution times may be substituted for migration distances and probability matrices constructed using retention or elution time windows. With any of these systems it is crucial to identify bracketing internal standards for the normalization of the patterns to eliminate variability due to day-to-day variations in the analytical technique (e.g. HPLC column drift as the column ages).

The authors thank R. C. Good for helpful discussions. They also thank M. A. Yakrus, W. R. Butler, C. L. Woodley and V. Silcox for providing the characterized Mycobacterium isolates.

\section{References}

Bascomb, S., Lapage, S. P., Curtis, M. A., \& WillcoX, W. R. (1973) Identification of bacteria by computer: identification of reference strains. Journal of General Microbiology 77, 291-315.

Butler, W. R., Jost, K. C. \& KILBURN, J. O. (1991). Identification of mycobacteria by high-performance liquid chromatography. Journal of Clinical Microbiology 29, 2468-2472.

DYBowsKI, W. \& FrankLIN, D. A. (1968). Conditional probability and the identification of bacteria: a pilot study. Journal of General Microbiology 54, 215-229.

Friedman, R. B., Bruce D., Maclowry J. \& Brenner, V. (1973) Computer-assisted identification of bacteria. Journal of Clinical Pathology 60, 395-403.

GOOD, R. C. \& SNIDER, D. E. (1982). Isolation of nontuberculosis mycobacteria in the United States, 1980. Journal of Infectious Diseases 146, 829-833.

JACKMAN, P. J. H. (1983). A program in BASIC for numerical taxonomy of micro-organisms based on electrophoretic band positions. Microbios Letters 23, 119-124.

KERSTERS, K. \& DE LEY, J. (1975). Identification and grouping of bacteria by numerical analysis of their electrophoretic protein patterns. Journal of General Microbiology 87, 333-342.

LAPAGe, S. P., Bascomb, S., WillcoX W. R. \& CuRTis, M. A. (1973). Identification of bacteria by computer: general aspects and perspectives. Journal of General Microbiology 77, 273-290.

Mullis, K. B. \& FaloONA, F. A. (1987). Specific synthesis of DNA in vitro via a polymerase-catalyzed chain reaction. Methods in Enzymology 155, 335-350.

Plikaytis, B. D., Carlone G. M. \& Plikaytis, B. B. (1986). Numerical analysis of normalized whole-cell protein profiles after sodium dodecyl sulphate-polyacrylamide gel electrophoresis. Journal of General Microbiology 132, 2653-2660.

Plikaytis, B. B., Plikaytis, B. D., Yakrus, M. A., Butler, W. R., Woodley, C. L., Silcox, V. A. \& ShinNick, T. M. (1992). Differentiation of slow growing Mycobacterium species including Mycobacterium tuberculosis by gene amplification and restriction fragment length polymorphism. Journal of Clinical Microbiology 30, $1815-1822$.

Shinnick, T. M., Sweetser, D., Thole, J., van Embden, J. D. A. \& Young, R. A. (1987). The etiologic agents of leprosy and tuberculosis share an immunoreactive protein antigen with the vaccine strain Mycobacterium bovis BCG. Infection and Immunity 55, 1932-1935.

SNeATH, P. H. A. \& Sokal, R. R. (1973). Numerical Taxonomy, The Principles and Practice of Numerical Classification, pp. 394-400. San Francisco: W. H. Freeman.

Wayne, L. G., Krichevsky, M. I., Portyrata, D. \& Jackson, C. K. (1984). Diagnostic probability matrix for identification of slowly growing mycobacteria in clinical laboratories. Journal of Clinical Microbiology 20, 722-729.

Willcox, W. R., Lapage, S. P., Bascomb, S. \& Curtis, M. A. (1973). Identification of bacteria by computer: theory and programming Journal of General Microbiology 77, 317-330. 